\title{
Optimal Replenishment Policy for Weibull-Distributed Deteriorating Items with Trapezoidal Demand Rate and Partial Backlogging
}

\author{
Lianxia Zhao \\ School of Management, Shanghai University, Shanghai 200444, China \\ Correspondence should be addressed to Lianxia Zhao; zhaolx2004@163.com
}

Received 2 December 2015; Accepted 27 March 2016

Academic Editor: Alireza Amirteimoori

Copyright (C) 2016 Lianxia Zhao. This is an open access article distributed under the Creative Commons Attribution License, which permits unrestricted use, distribution, and reproduction in any medium, provided the original work is properly cited.

An inventory model for Weibull-distributed deteriorating items is considered so as to minimize the total cost per unit time in this paper. The model starts with shortage, allowed partial backlogging, and trapezoidal demand rate. By analyzing the model, an efficient solution procedure is proposed to determine the optimal replenishment and the optimal order quantity and the average total costs are also obtained. Finally, numerical examples are provided to illustrate the theoretical results and a sensitivity analysis of the major parameters with respect to the stability of optimal solution is also carried out.

\section{Introduction}

Deteriorating is a general phenomenon for many products, in that fruits or vegetables are spoiled directly while alcohol undergoes physical depletion over time, and electronic products deteriorate through a gradual loss of potential utility as time passed. Based on the above situation, the effect of deteriorating for items can not be disregarded in many inventory systems. An inventory model with a constant decaying rate for items is proposed by Ghare and Schrader in 1963 [1], while Covert and Philip [2] extended Ghare and Schrader's model to an EOQ model with a two-parameter Weibull-distributed deteriorating rate. Since then, there are many researchers who focused on such topic; Wee et al. [3] considered an inventory model for deteriorating items with quantity discount, pricing, and partial backlogging, and more related articles can be referred to like Yang et al. [4], Shah et al. [5], Yang et al. [6], and so forth.

However, it is impossible for deteriorating items that the demand rate increases continuously during their full life cycle. Based on such reasons, Hill [7] proposed an inventory model with ramp-type demand rate, which extended to allow shortage proposed by Mandal and Pal [8]. Further,
Wu [9] extended Mandal and Pal's model to have Weibulldistributed deterioration and time-dependent backlogging. Skouri et al. [10] considered a model with general ramp-type demand rate, partial backlogging, and Weibull deterioration rate. Tan and Weng [11] considered a discrete inventory model for deteriorating items with partial backlogging. Pal et al. [12] considered a production inventory model for deteriorating items with ramp-type demand rate under the effect of inflation and shortages under fuzziness. There are many other related literatures about such inventory model, such as Ahmed et al. [13], Agrawal et al. [14], Singh and Pattnayak [15], Wu et al. [16], and Rabbani et al. [17].

While, for some short life cycle products, the demand rate may increase up to a certain level in the early stage of marketing, then reach a stabilized period, and finally decrease to zero and reach the end of their life cycle, Cheng and Wang [18] proposed an inventory model for deteriorating items with trapezoidal type demand rate. Further, Cheng et al. [19] extended the model to be of time-dependent deteriorating items with trapezoidal type demand rate and partial backlogging. Uthayakumar and Rameswari [20] studied a model for defective items with trapezoidal type demand rate to determine the optimal product reliability. Lin [21] and 


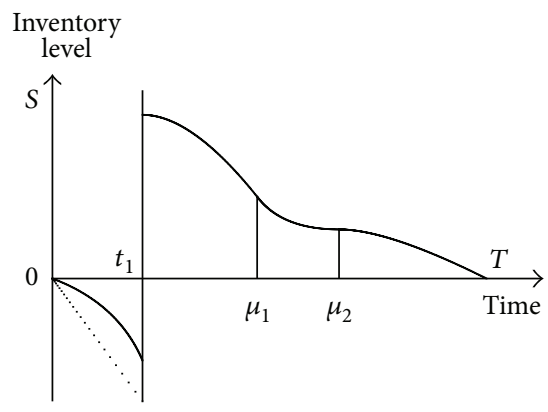

Case 1: $0<t_{1} \leq \mu_{1}$

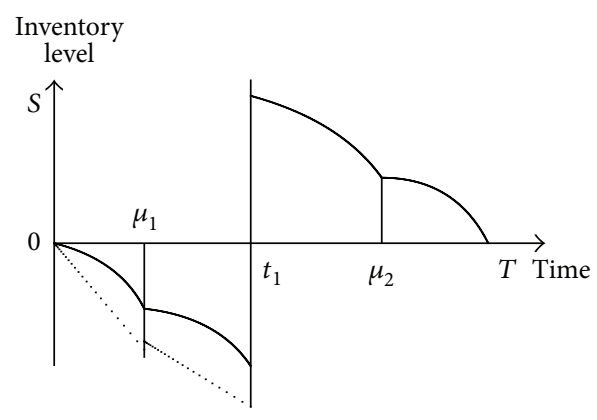

Case $2: \mu_{1} \leq t_{1} \leq \mu_{2}$

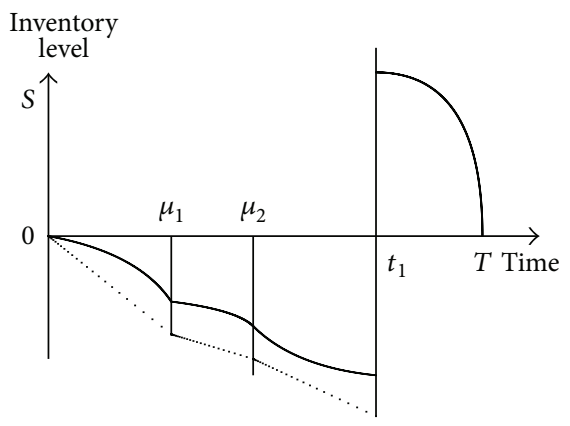

Case 3: $\mu_{2} \leq t_{1}<T$

FIGURE 1: Graphical representation of inventory level over the cycle starting with shortage.

Lin et al. [22] considered the inventory model which was proposed by Cheng and Wang [18]. Zhao [23] studied an inventory model for Weibull-distributed deterioration items with trapezoidal type demand rate and partial backlogging in which the model begins with no-shortage. While, in real situation, customers will respond differently in case of shortages, either to wait until replenishment or to look for alternative products, in this paper, we study an inventory model beginning with shortage, Weibull-distributed deterioration items, trapezoidal type demand rate, and time-dependent partial backlogging. By analyzing the studied model, we propose an optimal replenishment strategy for it and also carry out a sensitivity analysis of the main parameters.

The remainder of the paper is organized as follows. Notations and assumptions are described in Section 2. Section 3 constructs and analyzes the inventory model starting with shortage. Some numerical examples to illustrate the solution procedure are provided; then sensitivity analysis of the major parameters is also carried out in Section 4. Finally, a conclusion is presented.

\section{Notations and Assumptions}

The fundamental notations and assumptions used in this paper are given as follows.

(i) $I(t)$ is the inventory level at time $t, 0 \leq t \leq T$, where $T$ is the length of inventory cycle.

(ii) $t_{1}$ denotes the replenishment time, and $t_{1}^{*}$ denotes the optimal $t_{1}$.

(iii) $S$ is the maximum inventory level for each ordering cycle.

(iv) $Q^{*}$ denotes the optimal ordering quantity.

(v) $A_{0}$ denotes the fixed cost per order.

(vi) $c_{1}$ is the cost of each deteriorated item, $c_{2}$ is the unit inventory holding cost per unit time, $c_{3}$ is the unit shortage cost per unit time, and $c_{4}$ is the unit lost sales cost.

(vii) $\mathrm{C}_{i}\left(t_{1}\right), i=1,2,3$, denotes the average total cost per unit time under different conditions, respectively. (viii) $\mathrm{TC}\left(t_{1}\right)$ denotes the average total cost per unit time.

(ix) The demand rate, $D(t)$, which is positive and consecutive, is assumed to be a trapezoidal type function of time; that is,

$$
D(t)= \begin{cases}f(t), & t \leq \mu_{1}, \\ D_{0}, & \mu_{1}<t<\mu_{2}, \\ g(t), & \mu_{2} \leq t<T,\end{cases}
$$

where $\mu_{1}$ is time point changing from the increasing demand function $f(t)$ to the constant demand $D_{0}$, and $\mu_{2}$ is time point changing from the constant demand $D_{0}$ to the decreasing demand function $g(t)$.

(x) The replenishment rate is infinite; that is, replenishment is instantaneous.

(xi) The time to deterioration of the item is distributed as Weibull $(\alpha, \beta)$; that is, the deterioration rate is $\theta(t)=$ $\alpha \beta t^{\beta-1}$, where $\alpha$ is the scale parameter, $0<\alpha<1$, and $\beta$ is the shape parameter, $0<\beta$.

(xii) Shortage is allowed and let $e^{-\delta t}$ be fraction of shortages backlogged, where $t$ is the waiting time up to the next replenishment and $\delta$ is a tiny positive constant; that is, $\delta \ll 1 / T$.

\section{Inventory Model Starting with Shortage}

The system of model begins with shortage and until $t_{1}$ the shortage achieves its maximum; then replenishment occurs at time $t=t_{1}$. All of the shortage demand during $\left(0, t_{1}\right)$ is partial backlogged (Figure 1). At $t_{1}$, the total number of backlogged items will be satisfied by the replenishment and the inventory level up to $S$, while the deteriorating occurs during the time of $\left[t_{1}, T\right]$. According to the notations and 
assumptions mentioned above, the model is described as shown in Figure 1:

$$
\frac{d I(t)}{d t}= \begin{cases}-e^{-\delta\left(t_{1}-t\right)} D(t), & 0<t<t_{1}, \\ -\theta(t) I(t)-D(t), & t_{1}<t<T,\end{cases}
$$

with boundary condition $I(0)=0, I\left(t_{1}\right)=S, I(T)=0$.

Based on the values of $t_{1}, \mu_{1}$, and $\mu_{2}$, three possible cases are presented as follows.
Case $1\left(0<t_{1} \leq \mu_{1}\right)$. If the replenishment time $t_{1} \in\left(0, \mu_{1}\right]$, from (2), we have

$$
\frac{d I(t)}{d t}= \begin{cases}-e^{-\delta\left(t_{1}-t\right)} f(t), & 0<t<t_{1}, \\ -\alpha \beta t^{\beta-1} I(t)-f(t), & t_{1}<t<\mu_{1}, \\ -\alpha \beta t^{\beta-1} I(t)-D_{0}, & \mu_{1}<t<\mu_{2}, \\ -\alpha \beta t^{\beta-1} I(t)-g(t), & \mu_{2}<t<T .\end{cases}
$$

Solving the differential equations (3) with $I(0)=0$, we have

$$
I(t)= \begin{cases}-\int_{0}^{t} e^{\delta\left(x-t_{1}\right)} f(x) d x, & 0<t<t_{1}, \\ \int_{t}^{\mu_{1}} e^{\alpha\left(x^{\beta}-t^{\beta}\right)} f(x) d x+D_{0} \int_{\mu_{1}}^{\mu_{2}} e^{\alpha\left(x^{\beta}-t^{\beta}\right)} d x+\int_{\mu_{2}}^{T} e^{\alpha\left(x^{\beta}-t^{\beta}\right)} g(x) d x, & t_{1}<t<\mu_{1}, \\ D_{0} \int_{t}^{\mu_{2}} e^{\alpha\left(x^{\beta}-t^{\beta}\right)} d x+\int_{\mu_{2}}^{T} e^{\alpha\left(x^{\beta}-t^{\beta}\right)} g(x) d x, & \mu_{1}<t<\mu_{2}, \\ \int_{t}^{T} e^{\alpha\left(x^{\beta}-t^{\beta}\right)} g(x) d x, & \mu_{2}<t<T .\end{cases}
$$

The total replenishment quantity can be computed as

$$
\begin{aligned}
S= & I\left(t_{1}\right) \\
= & \int_{t_{1}}^{\mu_{1}} e^{\alpha\left(x^{\beta}-t_{1}^{\beta}\right)} f(x) d x+D_{0} \int_{\mu_{1}}^{\mu_{2}} e^{\alpha\left(x^{\beta}-t_{1}^{\beta}\right)} d x \\
& +\int_{\mu_{2}}^{T} e^{\alpha\left(x^{\beta}-t_{1}^{\beta}\right)} g(x) d x .
\end{aligned}
$$

The total shortage quantity $B_{T}$ during the interval $\left[0, t_{1}\right)$ is

$$
B_{T}=\int_{0}^{t_{1}}\left(t_{1}-t\right) e^{\delta\left(t-t_{1}\right)} f(t) d t
$$

The total number of perished items during the interval $\left[t_{1}, T\right]$, say $D_{T}$, is

$$
\begin{aligned}
D_{T}= & \int_{t_{1}}^{\mu_{1}}\left(e^{\alpha\left(t^{\beta}-t_{1}^{\beta}\right)}-1\right) f(t) d t \\
& +D_{0} \int_{\mu_{1}}^{\mu_{2}}\left(e^{\alpha\left(t^{\beta}-t_{1}^{\beta}\right)}-1\right) d t \\
& +\int_{\mu_{2}}^{T}\left(e^{\alpha\left(t^{\beta}-t_{1}^{\beta}\right)}-1\right) g(t) d t .
\end{aligned}
$$

The total number of inventories carried during the interval $\left[t_{1}, T\right]$, say $H_{T}$, is

$$
\begin{aligned}
H_{T}= & \int_{t_{1}}^{\mu_{1}} \int_{t}^{\mu_{1}} e^{\alpha\left(x^{\beta}-t^{\beta}\right)} f(x) d x d t \\
& +D_{0} \int_{t_{1}}^{\mu_{1}} \int_{\mu_{1}}^{\mu_{2}} e^{\alpha\left(x^{\beta}-t^{\beta}\right)} d x d t
\end{aligned}
$$

$$
\begin{aligned}
& +\int_{t_{1}}^{\mu_{2}} \int_{\mu_{2}}^{T} e^{\alpha\left(x^{\beta}-t^{\beta}\right)} g(x) d x d t \\
& +D_{0} \int_{\mu_{1}}^{\mu_{2}} \int_{t}^{\mu_{2}} e^{\alpha\left(x^{\beta}-t^{\beta}\right)} d x d t \\
& +\int_{\mu_{2}}^{T} \int_{t}^{T} e^{\alpha\left(x^{\beta}-t^{\beta}\right)} g(x) d x d t .
\end{aligned}
$$

The total of lost sales during the interval $\left[0, t_{1}\right]$, say $L_{T}$, is

$$
L_{T}=\int_{0}^{t_{1}}\left(1-e^{\delta\left(t-t_{1}\right)}\right) f(t) d t
$$

Therefore, the average total cost per unit time under the condition $t_{1} \leq \mu_{1}$ can be given by

$$
\begin{aligned}
& \mathrm{C}_{1}\left(t_{1}\right)=\frac{1}{T}\left[A_{0}+\mathrm{c}_{1} D_{T}+\mathrm{c}_{2} H_{T}+\mathrm{c}_{3} B_{T}+\mathrm{c}_{4} L_{T}\right] \\
& =\frac{1}{T}\left\{A_{0}+\mathrm{c}_{3}\left[\int_{0}^{t_{1}}\left(t_{1}-t\right) e^{\delta\left(t-t_{1}\right)} f(t) d t\right]\right. \\
& +\mathrm{c}_{4}\left[\int_{0}^{t_{1}}\left(1-e^{\delta\left(t-t_{1}\right)}\right) f(t) d t\right] \\
& +\mathrm{c}_{1}\left[\int_{t_{1}}^{\mu_{1}}\left(e^{\alpha\left(t^{\beta}-t_{1}^{\beta}\right)}-1\right) f(t) d t\right. \\
& +D_{0} \int_{\mu_{1}}^{\mu_{2}}\left(e^{\alpha\left(t^{\beta}-t_{1}^{\beta}\right)}-1\right) d t
\end{aligned}
$$




$$
\begin{aligned}
& \left.+\int_{\mu_{2}}^{T}\left(e^{\alpha\left(t^{\beta}-t_{1}^{\beta}\right)}-1\right) g(t) d t\right] \\
& +c_{2}\left[\int_{t_{1}}^{\mu_{1}} \int_{t}^{\mu_{1}} e^{\alpha\left(x^{\beta}-t^{\beta}\right)} f(x) d x d t\right. \\
& +D_{0} \int_{t_{1}}^{\mu_{1}} \int_{\mu_{1}}^{\mu_{2}} e^{\alpha\left(x^{\beta}-t^{\beta}\right)} d x d t \\
& +\int_{t_{1}}^{\mu_{2}} \int_{\mu_{2}}^{T} e^{\alpha\left(x^{\beta}-t^{\beta}\right)} g(x) d x d t \\
& +D_{0} \int_{\mu_{1}}^{\mu_{2}} \int_{t}^{\mu_{2}} e^{\alpha\left(x^{\beta}-t^{\beta}\right)} d x d t \\
& \left.\left.+\int_{\mu_{2}}^{T} \int_{t}^{T} e^{\alpha\left(x^{\beta}-t^{\beta}\right)} g(x) d x d t\right]\right\} .
\end{aligned}
$$

Taking the first and the second order derivative of $\mathrm{C}_{1}\left(t_{1}\right)$ with respect to $t_{1}$, respectively, we have

$$
\begin{aligned}
& \frac{d \mathrm{C}_{1}\left(t_{1}\right)}{d t_{1}}=\frac{1}{T}\left\{\mathrm{c}_{3} \int_{0}^{t_{1}}\left(1-\delta t_{1}+\delta t\right) e^{\delta\left(t-t_{1}\right)} f(t) d t\right. \\
& +\mathrm{c}_{4} \delta \int_{0}^{t_{1}} e^{\delta\left(t-t_{1}\right)} f(t) d t-\left(\mathrm{c}_{2}+\mathrm{c}_{1} \alpha \beta t_{1}^{\beta-1}\right) \\
& \left.\cdot \int_{t_{1}}^{T} e^{\alpha\left(t^{\beta}-t_{1}^{\beta}\right)} D(t) d t\right\}, \\
& \frac{d^{2} \mathrm{C}_{1}\left(t_{1}\right)}{d t_{1}^{2}}=\frac{1}{T}\left\{\mathrm { c } _ { 3 } \left[f\left(t_{1}\right)\right.\right. \\
& \left.\quad+\delta \int_{0}^{t_{1}}\left(\delta t_{1}-\delta t-2\right) e^{\delta\left(t-t_{1}\right)} f(t) d t\right]+\mathrm{c}_{4} \delta\left[f\left(t_{1}\right)\right. \\
& \left.\quad-\delta \int_{0}^{t_{1}} e^{\delta\left(t-t_{1}\right)} f(t) d t\right]+\left(\mathrm{c}_{1} \alpha \beta t_{1}^{\beta-1}+\mathrm{c}_{2}\right) f\left(t_{1}\right) \\
& \quad+\alpha \beta t_{1}^{\beta-2}\left[\mathrm{c}_{1}\left(\alpha \beta t_{1}^{\beta}-\beta+1\right)+\mathrm{c}_{2} t_{1}\right] \\
& \left.\quad \cdot \int_{t_{1}}^{T} e^{\alpha\left(t^{\beta}-t_{1}^{\beta}\right)} D(t) d t\right\} .
\end{aligned}
$$

Then, we have

$$
\left.\frac{d \mathrm{C}_{1}\left(t_{1}\right)}{d t_{1}}\right|_{t_{1}=0}=-\frac{\mathrm{c}_{2}}{T} \int_{0}^{T} e^{\alpha t^{\beta}} D(t) d t<0,
$$

and let

$$
\begin{aligned}
\Delta_{1} & =c_{3} \int_{0}^{\mu_{1}}\left(1-\delta \mu_{1}+\delta t\right) e^{\delta\left(t-\mu_{1}\right)} f(t) d t \\
& +c_{4} \delta \int_{0}^{\mu_{1}} e^{\delta\left(t-\mu_{1}\right)} f(t) d t-\left(c_{2}+c_{1} \alpha \beta \mu_{1}^{\beta-1}\right) \\
& \cdot\left(D_{0} \int_{\mu_{1}}^{\mu_{2}} e^{\alpha\left(t^{\beta}-\mu_{1}^{\beta}\right)} d t+\int_{\mu_{2}}^{T} e^{\alpha\left(t^{\beta}-\mu_{1}^{\beta}\right)} g(t) d t\right) .
\end{aligned}
$$

Therefore, (i) if $\Delta_{1}>0$ and $d^{2} \mathrm{C}_{1}\left(t_{1}\right) / d t_{1}^{2}>0$, there exists a unique solution $t_{1}^{*} \in\left(0, \mu_{1}\right)$ satisfying $d \mathrm{C}_{1}\left(t_{1}\right) / d t_{1}=0$, and $\mathrm{C}_{1}\left(t_{1}\right)$ obtains its minimum at $t_{1}=t_{1}^{*}$; (ii) if $\Delta_{1}<0$ and $d^{2} \mathrm{C}_{1}\left(t_{1}\right) / d t_{1}^{2}>0$, it means that $\mathrm{C}_{1}\left(t_{1}\right)$ is a nonincreasing function and obtains its minimum value at point $t_{1}^{*}=\mu_{1}$; (iii) if the sign of $d^{2} \mathrm{C}_{1}\left(t_{1}\right) / d t_{1}^{2}$ is indefinite, then the curve of $\mathrm{C}_{1}\left(t_{1}\right)$ is $\mathrm{W}$-shape, and $\mathrm{C}_{1}\left(t_{1}\right)$ may obtain its minimum at more than one point; without loss of generality, set $t_{1}=t_{1}^{*}$, where $t_{1}^{*}$ is one of the solutions of $d \mathrm{C}_{1}\left(t_{1}\right) / d t_{1}=0$.

Thus, the optimal value of the order level, $S=I\left(t_{1}^{*}\right)$, is

$$
\begin{aligned}
S^{*}= & \int_{t_{1}^{*}}^{\mu_{1}} e^{\alpha\left(x^{\beta}-t_{1}^{* \beta}\right)} f(x) d x+D_{0} \int_{\mu_{1}}^{\mu_{2}} e^{\alpha\left(x^{\beta}-t_{1}^{* \beta}\right)} d x \\
& +\int_{\mu_{2}}^{T} e^{\alpha\left(x^{\beta}-t_{1}^{* \beta}\right)} g(x) d x,
\end{aligned}
$$

and the optimal order quantity $Q^{*}$ is

$$
Q^{*}=S^{*}+\int_{0}^{t_{1}^{*}} e^{\delta\left(t-t_{1}^{*}\right)} f(t) d t
$$

Case $2\left(\mu_{1} \leq t_{1} \leq \mu_{2}\right)$. If the replenishment time $t_{1} \in\left[\mu_{1}, \mu_{2}\right]$, then from (2), we have

$$
\frac{d I(t)}{d t}= \begin{cases}-e^{-\delta\left(t_{1}-t\right)} f(t), & 0<t<\mu_{1}, \\ -e^{-\delta\left(t_{1}-t\right)} D_{0}, & \mu_{1}<t<t_{1}, \\ -\alpha \beta t^{\beta-1} I(t)-D_{0}, & t_{1}<t<\mu_{2}, \\ -\alpha \beta t^{\beta-1} I(t)-g(t), & \mu_{2}<t<T .\end{cases}
$$

Solving the differential equation (17) with $I(0)=I(T)=0$, we have

$$
I(t)= \begin{cases}-\int_{0}^{t} e^{\delta\left(x-t_{1}\right)} f(x) d x, & 0<t<\mu_{1}, \\ -\frac{D_{0}}{\delta}\left(e^{\delta\left(t-t_{1}\right)}-e^{\delta\left(\mu_{1}-t_{1}\right)}\right)-\int_{0}^{\mu_{1}} e^{\delta\left(x-t_{1}\right)} f(x) d x, & \mu_{1}<t<t_{1}, \\ D_{0} \int_{t}^{\mu_{2}} e^{\alpha\left(x^{\beta}-t^{\beta}\right)} d x+\int_{\mu_{2}}^{T} e^{\alpha\left(x^{\beta}-t^{\beta}\right)} g(x) d x, & t_{1}<t<\mu_{2}, \\ \int_{t}^{T} e^{\alpha\left(x^{\beta}-t^{\beta}\right)} g(x) d x, & \mu_{2}<t<T .\end{cases}
$$


From (18), the total replenishment quantity can be computed:

$$
S=I\left(t_{1}\right)=D_{0} \int_{t_{1}}^{\mu_{2}} e^{\alpha\left(x^{\beta}-t_{1}^{\beta}\right)} d x+\int_{\mu_{2}}^{T} e^{\alpha\left(x^{\beta}-t_{1}^{\beta}\right)} g(x) d x .
$$

The total shortage quantity during the interval $\left(0, t_{1}\right]$ is

$$
\begin{aligned}
B_{T}= & \int_{0}^{\mu_{1}}\left(t_{1}-t\right) e^{\delta\left(t-t_{1}\right)} f(t) d t \\
& +\frac{D_{0}}{\delta} \int_{\mu_{1}}^{t_{1}}\left(e^{\delta\left(t-t_{1}\right)}-e^{\delta\left(\mu_{1}-t_{1}\right)}\right) d t .
\end{aligned}
$$

The total number of perished items in the interval $\left[t_{1}, T\right]$ is

$$
\begin{aligned}
D_{T}= & D_{0} \int_{t_{1}}^{\mu_{2}}\left(e^{\alpha\left(t^{\beta}-t_{1}^{\beta}\right)}-1\right) d t \\
& +\int_{\mu_{2}}^{T}\left(e^{\alpha\left(t^{\beta}-t_{1}^{\beta}\right)}-1\right) g(t) d t .
\end{aligned}
$$

The total number of inventories carried during the interval $\left[t_{1}, T\right]$ is

$$
\begin{aligned}
H_{T}= & D_{0} \int_{t_{1}}^{\mu_{2}} \int_{t}^{\mu_{2}} e^{\alpha\left(x^{\beta}-t^{\beta}\right)} d x d t \\
& +\int_{t_{1}}^{\mu_{2}} \int_{\mu_{2}}^{T} e^{\alpha\left(x^{\beta}-t^{\beta}\right)} g(x) d x d t \\
& +\int_{\mu_{2}}^{T} \int_{t}^{T} e^{\alpha\left(x^{\beta}-t^{\beta}\right)} g(x) d x d t .
\end{aligned}
$$

The total of lost sales during the interval $\left[0, t_{1}\right]$ is

$$
\begin{aligned}
L_{T}= & \int_{0}^{\mu_{1}}\left(1-e^{\delta\left(t-t_{1}\right)}\right) f(t) d t \\
& +D_{0} \int_{\mu_{1}}^{t_{1}}\left(1-e^{\delta\left(t-t_{1}\right)}\right) d t .
\end{aligned}
$$

Therefore, the average total cost per unit time under the condition $\mu_{1}<t_{1} \leq \mu_{2}$ can be given by

$$
\begin{aligned}
& \mathrm{C}_{2}\left(t_{1}\right)=\frac{1}{T}\left[A_{0}+c_{1} D_{T}+c_{2} H_{T}+c_{3} B_{T}+c_{4} L_{T}\right] \\
& \quad=\frac{1}{T}\left\{A_{0}+c_{1}\left[D_{0} \int_{t_{1}}^{\mu_{2}}\left(e^{\alpha\left(t^{\beta}-t_{1}^{\beta}\right)}-1\right) d t\right.\right. \\
& \left.+\int_{\mu_{2}}^{T}\left(e^{\alpha\left(t^{\beta}-t_{1}^{\beta}\right)}-1\right) g(t) d t\right] \\
& +c_{2}\left[D_{0} \int_{t_{1}}^{\mu_{2}} \int_{t}^{\mu_{2}} e^{\alpha\left(x^{\beta}-t^{\beta}\right)} d x d t\right. \\
& +\int_{t_{1}}^{\mu_{2}} \int_{\mu_{2}}^{T} e^{\alpha\left(x^{\beta}-t^{\beta}\right)} g(x) d x d t \\
& \left.+\int_{\mu_{2}}^{T} \int_{t}^{T} e^{\alpha\left(x^{\beta}-t^{\beta}\right)} g(x) d x d t\right]
\end{aligned}
$$

$$
\begin{aligned}
& +\mathrm{c}_{4}\left[\int_{0}^{\mu_{1}}\left(1-e^{\delta\left(t-t_{1}\right)}\right) f(t) d t\right. \\
& \left.+D_{0} \int_{\mu_{1}}^{t_{1}}\left(1-e^{\delta\left(t-t_{1}\right)}\right) d t\right] \\
& +\mathrm{c}_{3}\left[\int_{0}^{\mu_{1}}\left(t_{1}-t\right) e^{\delta\left(t-t_{1}\right)} f(t) d t\right. \\
& \left.\left.+\frac{D_{0}}{\delta} \int_{\mu_{1}}^{t_{1}}\left(e^{\delta\left(t-t_{1}\right)}-e^{\delta\left(\mu_{1}-t_{1}\right)}\right) d t\right]\right\} .
\end{aligned}
$$

Taking the first and the second order derivative of $\mathrm{C}_{2}\left(t_{1}\right)$ with respect to $t_{1}$, respectively, we have

$$
\begin{aligned}
& \frac{d \mathrm{C}_{2}\left(t_{1}\right)}{d t_{1}}=\frac{1}{T}\left\{\mathrm { c } _ { 4 } \delta \left[\int_{0}^{\mu_{1}} e^{\delta\left(t-t_{1}\right)} f(t) d t\right.\right. \\
& \left.+D_{0} \int_{\mu_{1}}^{t_{1}} e^{\delta\left(t-t_{1}\right)} d t\right]-\left(c_{1} \alpha \beta t_{1}^{\beta-1}+c_{2}\right) \\
& \cdot\left[D_{0} \int_{t_{1}}^{\mu_{2}} e^{\alpha\left(t^{\beta}-t_{1}^{\beta}\right)} d t+\int_{\mu_{2}}^{T} e^{\alpha\left(t^{\beta}-t_{1}^{\beta}\right)} g(t) d t\right] \\
& +c_{3}\left[\int_{0}^{\mu_{1}}\left(1-\delta t_{1}+\delta t\right) e^{\delta\left(t-t_{1}\right)} f(t) d t\right. \\
& \left.\left.-D_{0}\left(\mu_{1}-t_{1}\right) e^{\delta\left(\mu_{1}-t_{1}\right)}\right]\right\} \text {, } \\
& \frac{d^{2} \mathrm{C}_{2}\left(t_{1}\right)}{d t_{1}^{2}}=\frac{1}{T}\left\{\alpha \beta t_{1}^{\beta-2}\left(\mathrm{c}_{1}\left(\alpha \beta t_{1}^{\beta}-\beta+1\right)+\mathrm{c}_{2} t_{1}\right)\right. \\
& \cdot\left[D_{0} \int_{t_{1}}^{\mu_{2}} e^{\alpha\left(t^{\beta}-t_{1}^{\beta}\right)} d t+\int_{\mu_{2}}^{T} e^{\alpha\left(t^{\beta}-t_{1}^{\beta}\right)} g(t) d t\right] \\
& +\left(c_{1} \alpha \beta t_{1}^{\beta-1}+c_{2}\right) D_{0}+c_{4} \delta\left[D_{0} e^{\delta\left(\mu_{1}-t_{1}\right)}\right. \\
& \left.-\delta \int_{0}^{\mu_{1}} e^{\delta\left(t-t_{1}\right)} f(t) d t\right] \\
& +c_{3}\left[\int_{0}^{\mu_{1}}\left(\delta^{2} t_{1}-\delta^{2} t-2 \delta\right) e^{\delta\left(t-t_{1}\right)} f(t) d t\right. \\
& \left.\left.+D_{0}\left(1+\delta \mu_{1}-\delta t_{1}\right) e^{\delta\left(\mu_{1}-t_{1}\right)}\right]\right\} \text {. }
\end{aligned}
$$

Let

$$
\begin{aligned}
\Delta_{2} & =c_{3}\left[\int_{0}^{\mu_{1}}\left(1-\delta \mu_{2}+\delta t\right) e^{\delta\left(t-\mu_{2}\right)} f(t) d t\right. \\
& \left.+D_{0}\left(\mu_{2}-\mu_{1}\right) e^{\delta\left(\mu_{1}-\mu_{2}\right)}\right] \\
& +c_{4} \delta\left[\int_{0}^{\mu_{1}} e^{\delta\left(t-\mu_{2}\right)} f(t) d t+D_{0} \int_{\mu_{1}}^{\mu_{2}} e^{\delta\left(t-\mu_{2}\right)} d t\right] \\
& -\left(c_{2}+c_{1} \alpha \beta \mu_{2}^{\beta-1}\right) \int_{\mu_{2}}^{T} e^{\alpha\left(t^{\beta}-\mu_{2}^{\beta}\right)} g(t) d t .
\end{aligned}
$$


Therefore, (i) if $\Delta_{1}<0, \Delta_{2}>0$, and $d^{2} \mathrm{C}_{2}\left(t_{1}\right) / d t_{1}^{2}>0$, there exists a unique solution $t_{1}^{*} \in\left(\mu_{1}, \mu_{2}\right)$ satisfying $d \mathrm{C}_{2}\left(t_{1}\right) / d t_{1}=$ 0 , and $\mathrm{C}_{2}\left(t_{1}\right)$ obtains its minimum at $t_{1}=t_{1}^{*}$; (ii) if $\Delta_{1}<$ $0, \Delta_{2} \leq 0$, and $d^{2} \mathrm{C}_{2}\left(t_{1}\right) / d t_{1}^{2}>0$, it means that $\mathrm{C}_{2}\left(t_{1}\right)$ is a nonincreasing function and obtains its minimum at point $t_{1}^{*}=\mu_{2}$; (iii) if $\Delta_{1} \geq 0, \Delta_{2}>0$, and $d^{2} \mathrm{C}_{2}\left(t_{1}\right) / d t_{1}^{2}>0$, it means that $\mathrm{C}_{2}\left(t_{1}\right)$ is a nondecreasing function and obtains its minimum at point $t_{1}^{*}=\mu_{1}$; (iv) if the sign of $d^{2} \mathrm{C}_{2}\left(t_{1}\right) / d t_{1}^{2}$ is indefinite, then the curve of $\mathrm{C}_{2}\left(t_{1}\right)$ is $\mathrm{W}$-shape, and $\mathrm{C}_{2}\left(t_{1}\right)$ may obtain its minimum value at more than one point; without loss of generality, set $t_{1}=t_{1}^{*}$, where $t_{1}^{*}$ is one of the solutions of $d \mathrm{C}_{2}\left(t_{1}\right) / d t_{1}=0$.

Thus, the optimal value of the order level, $S=I\left(t_{1}^{*}\right)$, is

$$
S^{*}=D_{0} \int_{t_{1}^{*}}^{\mu_{2}} e^{\alpha\left(t^{\beta}-t_{1}^{* \beta}\right)} d t+\int_{\mu_{2}}^{T} e^{\alpha\left(t^{\beta}-t_{1}^{* \beta}\right)} g(t) d t
$$

and the optimal order quantity $Q^{*}$ is

$$
Q^{*}=S^{*}+\int_{0}^{\mu_{1}} e^{\delta\left(t-t_{1}^{*}\right)} f(t) d t+D_{0} \int_{\mu_{1}}^{t_{1}^{*}} e^{\delta\left(t-t_{1}^{*}\right)} d t
$$

Case $3\left(\mu_{2} \leq t_{1}<T\right)$. If the replenishment time $t_{1} \in\left[\mu_{2}, T\right)$, from (2), we have

$$
\frac{d I(t)}{d t}= \begin{cases}-e^{-\delta\left(t_{1}-t\right)} f(t), & 0<t<\mu_{1}, \\ -e^{-\delta\left(t_{1}-t\right)} D_{0}, & \mu_{1}<t<\mu_{2}, \\ -e^{-\delta\left(t_{1}-t\right)} g(t), & \mu_{2}<t<t_{1}, \\ -\alpha \beta t^{\beta-1} I(t)-g(t), & t_{1}<t<T .\end{cases}
$$

Solving (30) with $I(0)=I(T)=0$, we have

$$
I(t)= \begin{cases}-\int_{0}^{t} e^{\delta\left(x-t_{1}\right)} f(x) d x, & 0<t<\mu_{1}, \\ \frac{D_{0}}{\delta}\left(e^{\delta\left(\mu_{1}-t_{1}\right)}-e^{\delta\left(t-t_{1}\right)}\right)-\int_{0}^{\mu_{1}} e^{\delta\left(x-t_{1}\right)} f(x) d x, & \mu_{1}<t<\mu_{2}, \\ -\int_{\mu_{2}}^{t} e^{\delta\left(x-t_{1}\right)} g(x) d x+\frac{D_{0}}{\delta}\left(e^{\delta\left(\mu_{1}-t_{1}\right)}-e^{\delta\left(\mu_{2}-t_{1}\right)}\right)-\int_{0}^{\mu_{1}} e^{\delta\left(x-t_{1}\right)} f(x) d x, & \mu_{2}<t<t_{1}, \\ \int_{t}^{T} e^{\alpha\left(x^{\beta}-t^{\beta}\right)} g(x) d x, & t_{1}<t<T .\end{cases}
$$

From the last equation of (31), the total replenishment quantity can be computed:

$$
S=I\left(t_{1}\right)=\int_{t_{1}}^{T} e^{\alpha\left(x^{\beta}-t_{1}^{\beta}\right)} g(x) d x .
$$

The total shortage quantity during the interval $\left(0, t_{1}\right]$ is

$$
\begin{aligned}
B_{T}= & \int_{0}^{\mu_{1}}\left(t_{1}-t\right) e^{\delta\left(t-t_{1}\right)} f(t) d t \\
& +\int_{\mu_{2}}^{t_{1}}\left(t_{1}-t\right) e^{\delta\left(t-t_{1}\right)} g(t) d t \\
& +\frac{D_{0}}{\delta^{2}}\left(e^{\delta\left(\mu_{1}-t_{1}\right)}-e^{\delta\left(\mu_{2}-t_{1}\right)}\right) \\
& +\frac{D_{0}}{\delta}\left[\left(t_{1}-\mu_{1}\right) e^{\delta\left(\mu_{1}-t_{1}\right)}-\left(t_{1}-\mu_{2}\right) e^{\delta\left(\mu_{2}-t_{1}\right)}\right] .
\end{aligned}
$$

The total number of perished items in the interval $\left[t_{1}, T\right]$ is

$$
D_{T}=\int_{t_{1}}^{T}\left(e^{\alpha\left(x^{\beta}-t_{1}^{\beta}\right)}-1\right) g(x) d x .
$$

The total number of inventories carried during the interval $\left[t_{1}, T\right]$ is

$$
H_{T}=\int_{t_{1}}^{T} \int_{t}^{T} e^{\alpha\left(x^{\beta}-t^{\beta}\right)} g(x) d x d t
$$

The total of lost sales during the interval $\left[0, t_{1}\right]$ is

$$
\begin{aligned}
L_{T}= & \int_{0}^{\mu_{1}}\left(1-e^{\delta\left(t-t_{1}\right)}\right) f(t) d t \\
& +D_{0} \int_{\mu_{1}}^{\mu_{2}}\left(1-e^{\delta\left(t-t_{1}\right)}\right) d t \\
& +\int_{\mu_{2}}^{t_{1}}\left(1-e^{\delta\left(t-t_{1}\right)}\right) g(t) d t .
\end{aligned}
$$

Therefore, the average total cost per unit time under the condition $\mu_{2}<t_{1} \leq T$ can be given by

$$
\begin{aligned}
& \mathrm{C}_{3}\left(t_{1}\right)=\frac{1}{T}\left[A_{0}+\mathrm{c}_{1} D_{T}+\mathrm{c}_{2} H_{T}+\mathrm{c}_{3} B_{T}+\mathrm{c}_{4} L_{T}\right] \\
& =\frac{1}{T}\left\{A_{0}+\mathrm{c}_{1} \int_{t_{1}}^{T}\left(e^{\alpha\left(x^{\beta}-t_{1}^{\beta}\right)}-1\right) g(x) d x\right. \\
& +\mathrm{c}_{2} \int_{t_{1}}^{T} \int_{t}^{T} e^{\alpha\left(x^{\beta}-t^{\beta}\right)} g(x) d x d t \\
& \quad+\mathrm{c}_{4}\left[\int_{0}^{\mu_{1}}\left(1-e^{\delta\left(t-t_{1}\right)}\right) f(t) d t\right. \\
& \quad+D_{0} \int_{\mu_{1}}^{\mu_{2}}\left(1-e^{\delta\left(t-t_{1}\right)}\right) d t
\end{aligned}
$$




$$
\begin{aligned}
& \left.+\int_{\mu_{2}}^{t_{1}}\left(1-e^{\delta\left(t-t_{1}\right)}\right) g(t) d t\right] \\
& +c_{3}\left[\int_{0}^{\mu_{1}}\left(t_{1}-t\right) e^{\delta\left(t-t_{1}\right)} f(t) d t\right. \\
& +\int_{\mu_{2}}^{t_{1}}\left(t_{1}-t\right) e^{\delta\left(t-t_{1}\right)} g(t) d t \\
& +\frac{D_{0}}{\delta^{2}}\left(e^{\delta\left(\mu_{1}-t_{1}\right)}-e^{\delta\left(\mu_{2}-t_{1}\right)}\right) \\
& \left.\left.+\frac{D_{0}}{\delta}\left(\left(t_{1}-\mu_{1}\right) e^{\delta\left(\mu_{1}-t_{1}\right)}-\left(t_{1}-\mu_{2}\right) e^{\delta\left(\mu_{2}-t_{1}\right)}\right)\right]\right\} .
\end{aligned}
$$

Taking the first and the second order derivative of $\mathrm{C}_{3}\left(t_{1}\right)$ with respect to $t_{1}$, respectively, we have

$$
\begin{aligned}
& \frac{d \mathrm{C}_{3}\left(t_{1}\right)}{d t_{1}}=\frac{1}{T}\left\{-\left(\mathrm{c}_{2}+\mathrm{c}_{1} \alpha \beta t_{1}^{\beta-1}\right) \int_{t_{1}}^{T} e^{\alpha\left(t^{\beta}-t_{1}^{\beta}\right)} g(t) d t\right. \\
& +\mathrm{c}_{4} \delta\left[\int_{0}^{\mu_{1}} e^{\delta\left(t-t_{1}\right)} f(t) d t+D_{0} \int_{\mu_{1}}^{\mu_{2}} e^{\delta\left(t-t_{1}\right)} d t\right. \\
& \left.+\int_{\mu_{2}}^{t_{1}} e^{\delta\left(t-t_{1}\right)} g(t) d t\right] \\
& +\mathrm{c}_{3}\left[\int_{0}^{\mu_{1}}\left(1+\delta t-\delta t_{1}\right) e^{\delta\left(t-t_{1}\right)} f(t) d t\right. \\
& +\int_{\mu_{2}}^{t_{1}}\left(1+\delta t-\delta t_{1}\right) e^{\delta\left(t-t_{1}\right)} g(t) d t \\
& \left.\left.+D_{0}\left(\left(t_{1}-\mu_{1}\right) e^{\delta\left(\mu_{1}-t_{1}\right)}-\left(t_{1}-\mu_{2}\right) e^{\delta\left(\mu_{2}-t_{1}\right)}\right)\right]\right\}
\end{aligned}
$$$$
\frac{d^{2} \mathrm{C}_{3}\left(t_{1}\right)}{d t_{1}^{2}}=\frac{1}{T}\left\{\alpha \beta t_{1}^{\beta-2}\left[\mathrm{c}_{1}\left(\alpha \beta t_{1}^{\beta}-\beta+1\right)+\mathrm{c}_{2} t_{1}\right]\right.
$$$$
\cdot \int_{t_{1}}^{T} e^{\alpha\left(t^{\beta}-t_{1}^{\beta}\right)} g(t) d t+\left(c_{2}+c_{1} \alpha \beta t_{1}^{\beta-1}\right) g\left(t_{1}\right)
$$$$
+\mathrm{c}_{4} \delta\left[\delta \int_{0}^{\mu_{1}} e^{\delta\left(t-t_{1}\right)} f(t) d t+D_{0} \delta \int_{\mu_{1}}^{\mu_{2}} e^{\delta\left(t-t_{1}\right)} d t\right.
$$$$
\left.+\delta \int_{\mu_{2}}^{t_{1}} e^{\delta\left(t-t_{1}\right)} g(t) d t+g\left(t_{1}\right)\right]
$$$$
+c_{2}\left[\alpha \beta t_{1}^{\beta-1} \int_{t_{1}}^{T} e^{\alpha\left(t^{\beta}-t_{1}^{\beta}\right)} g(t) d t+g\left(t_{1}\right)\right]
$$$$
+c_{3}\left[\int_{0}^{\mu_{1}}\left(\delta^{2} t_{1}-\delta^{2} t-2 \delta\right) e^{\delta\left(t-t_{1}\right)} f(t) d t\right.
$$$$
+\int_{\mu_{2}}^{t_{1}}\left(\delta^{2} t_{1}-\delta^{2} t-2 \delta\right) e^{\delta\left(t-t_{1}\right)} g(t) d t+g\left(t_{1}\right)
$$

$$
\begin{aligned}
& +D_{0}\left(\left(1+\delta \mu_{1}-\delta t_{1}\right) e^{\delta\left(\mu_{1}-t_{1}\right)}\right. \\
& \left.\left.\left.-\left(1+\delta \mu_{2}-\delta t_{1}\right) e^{\delta\left(\mu_{2}-t_{1}\right)}\right)\right]\right\}
\end{aligned}
$$

We know that

$$
\begin{aligned}
& \left.\frac{d \mathrm{C}_{3}\left(t_{1}\right)}{d t_{1}}\right|_{t_{1}=T}=\frac{1}{T}\left\{\mathrm { c } _ { 4 } \delta \left[\int_{0}^{\mu_{1}} e^{\delta(t-T)} f(t) d t\right.\right. \\
& \left.+D_{0} \int_{\mu_{1}}^{\mu_{2}} e^{\delta(t-T)} d t+\int_{\mu_{2}}^{T} e^{\delta(t-T)} g(t) d t\right] \\
& +c_{3}\left[\int_{0}^{\mu_{1}}(1+\delta t-\delta T) e^{\delta(t-T)} f(t) d t\right. \\
& +\int_{\mu_{2}}^{T}(1+\delta t-\delta T) e^{\delta(t-T)} g(t) d t \\
& \left.\left.+D_{0}\left(\left(T-\mu_{1}\right) e^{\delta\left(\mu_{1}-T\right)}-\left(T-\mu_{2}\right) e^{\delta\left(\mu_{2}-T\right)}\right)\right]\right\} \\
& >0
\end{aligned}
$$

Therefore, (i) if $\Delta_{2}<0$ and $d^{2} \mathrm{C}_{3}\left(t_{1}\right) / d t_{1}^{2}>0$, there exists a unique solution $t_{1}^{*} \in\left(\mu_{2}, T\right)$ satisfying $d \mathrm{C}_{3}\left(t_{1}\right) / d t_{1}=0$, and $\mathrm{C}_{3}\left(t_{1}\right)$ obtains its minimum at $t_{1}=t_{1}^{*}$; (ii) if $\Delta_{2} \geq 0$ and $d^{2} \mathrm{C}_{3}\left(t_{1}\right) / d t_{1}^{2}>0$, it means that $\mathrm{C}_{3}\left(t_{1}\right)$ is a nondecreasing function and obtains its minimum value at point $t_{1}^{*}=\mu_{2}$; (iii) if the sign of $d^{2} C_{3}\left(t_{1}\right) / d t_{1}^{2}$ is indefinite, then the curve of $\mathrm{C}_{3}\left(t_{1}\right)$ is $\mathrm{W}$-shape, and $\mathrm{C}_{3}\left(t_{1}\right)$ may obtain its minimum value at more than one point; without loss of generality, set $t_{1}=t_{1}^{*}$, where $t_{1}^{*}$ is one of the solutions of $d \mathrm{C}_{3}\left(t_{1}\right) / d t_{1}=0$. is

Therefore, the optimal value of the order level, $S=I\left(t_{1}^{*}\right)$,

$$
S^{*}=\int_{t_{1}^{*}}^{T} e^{\alpha\left(x^{\beta}-t_{1}^{* \beta}\right)} g(x) d x,
$$

and the optimal order quantity $Q^{*}$ is

$$
\begin{aligned}
Q^{*}= & S^{*}+\int_{0}^{\mu_{1}} e^{\delta\left(t-t_{1}^{*}\right)} f(t) d t+D_{0} \int_{\mu_{1}}^{\mu_{2}} e^{\delta\left(t-t_{1}^{*}\right)} d t \\
& +\int_{\mu_{2}}^{t_{1}^{*}} e^{\delta\left(t-t_{1}^{*}\right)} g(t) d t .
\end{aligned}
$$

From the above analysis, we obtain that the total average cost of the system over the interval $[0, T]$ is

$$
\mathrm{TC}\left(t_{1}\right)= \begin{cases}\mathrm{C}_{1}\left(t_{1}\right), & 0<t_{1} \leq \mu_{1}, \\ \mathrm{C}_{2}\left(t_{1}\right), & \mu_{1}<t_{1} \leq \mu_{2}, \\ \mathrm{C}_{3}\left(t_{1}\right), & \mu_{2}<t_{1}<T,\end{cases}
$$

where $\mathrm{C}_{1}\left(t_{1}\right), \mathrm{C}_{2}\left(t_{1}\right)$, and $\mathrm{C}_{3}\left(t_{1}\right)$ are obtained from (10), (24), and (37), respectively. 
Basing on the analyzed results above, we provide the results which ensure the existence of a unique $t_{1}$, say $t_{1}^{*}$, to minimize the total average cost for the model system starting with shortages. The procedure is as follows:

Step 0. Input the parameters, $\mu_{1}, \mu_{2}, T, a_{1}, a_{2}, b_{1}, \alpha, \beta, \delta$, $D_{0}$, and $\mathrm{c}_{i}, i=1,2,3,4$.

Step 1. Calculate $\Delta_{1}$ and $d^{2} \mathrm{C}_{1}\left(t_{1}\right) / d t_{1}^{2}$.

Step 1.1. If $\Delta_{1}>0$ and $d^{2} \mathrm{C}_{1}\left(t_{1}\right) / d t_{1}^{2}>0$, solve a unique $t_{1}^{*}$ from $d \mathrm{C}_{1}\left(t_{1}\right) / d t_{1}=0$, and the total average cost and the order quantity can be obtained by (10) and (16), respectively.

Step 1.2. If $\Delta_{1} \leq 0$ and $d^{2} \mathrm{C}_{1}\left(t_{1}\right) / d t_{1}^{2}>0$, then the optimal average cost and the optimal order quantity can be obtained by (10) and (16) at $t_{1}^{*}=\mu_{1}$, respectively.

Step 1.3. If the sign of $d^{2} \mathrm{C}_{1}\left(t_{1}\right) / d t_{1}^{2}$ is indefinite, then solve equation $d \mathrm{C}_{1}\left(t_{1}\right) / d t_{1}=0$. If the solution is not unique, by comparing their average costs $C_{1}\left(t_{1}\right)$ to obtain the optimal replenishment point, compute the order quantity by (16).

Step 2. Calculate $\Delta_{2}$ and $d^{2} \mathrm{C}_{2}\left(t_{1}\right) / d t_{1}^{2}$.

Step 2.1. If $\Delta_{1}<0, \Delta_{2}>0$, and $d^{2} \mathrm{C}_{2}\left(t_{1}\right) / d t_{1}^{2}>0$, then seek a unique $t_{1}^{*}$ from equation $d \mathrm{C}_{2}\left(t_{1}\right) / d t_{1}=0$, and the optimal total average cost and the optimal order quantity can be obtained by (24) and (29) at $t_{1}=t_{1}^{*}$, respectively.

Step 2.2. If $\Delta_{1}>0, \Delta_{2}>0$, and $d^{2} \mathrm{C}_{2}\left(t_{1}\right) / d t_{1}^{2}>0$, then $\mathrm{C}_{2}\left(t_{1}\right)$ obtains its minimum at $t_{1}^{*}=\mu_{1}$, and compute the order quantity by (29).

Step 2.3. If $\Delta_{1}<0, \Delta_{2} \leq 0$, and $d^{2} \mathrm{C}_{2}\left(t_{1}\right) / d t_{1}^{2}>0$, then $\mathrm{C}_{2}\left(t_{1}\right)$ obtains its minimum at $t_{1}^{*}=\mu_{2}$, and compute the order quantity by (29).

Step 2.4. If the sign of $d^{2} \mathrm{C}_{2}\left(t_{1}\right) / d t_{1}^{2}$ is indefinite, then solve equation $d \mathrm{C}_{2}\left(t_{1}\right) / d t_{1}=0$. If the solution is not unique, by comparing their average costs $\mathrm{C}_{2}\left(t_{1}\right)$ to obtain the optimal replenishment point, compute the optimal order quantity by (29).

\section{Step 3. Calculate $d^{2} \mathrm{C}_{3}\left(t_{1}\right) / d t_{1}^{2}$.}

Step 3.1. If $\Delta_{2}<0$ and $d^{2} \mathrm{C}_{3}\left(t_{1}\right) / d t_{1}^{2}>0$, seek the unique $t_{1}^{*}$ from equation $d \mathrm{C}_{3}\left(t_{1}\right) / d t_{1}=0$, and the total average cost and the order quantity can be obtained by (37) and (42), respectively.

Step 3.2. If $\Delta_{2} \geq 0$ and $d^{2} \mathrm{C}_{3}\left(t_{1}\right) / d t_{1}^{2}>0$, then $\mathrm{C}_{3}\left(t_{1}\right)$ obtains its minimum at $t_{1}^{*}=\mu_{2}$, and compute the order quantity by (42).

Step 3.3. If the sign of $d^{2} \mathrm{C}_{3}\left(t_{1}\right) / d t_{1}^{2}$ is indefinite, then solve equation $d \mathrm{C}_{3}\left(t_{1}\right) / d t_{1}=0$. If the solution is not unique, by comparing their average costs $C_{3}\left(t_{1}\right)$ to obtain the optimal replenishment point, compute the order quantity by (42).
Step 4. Find $\mathrm{TC}\left(t_{1}^{*}\right)=\min \left\{\mathrm{C}_{1}\left(t_{1}^{*}\right), \mathrm{C}_{2}\left(t_{1}^{*}\right), \mathrm{C}_{3}\left(t_{1}^{*}\right)\right\}$ and accordingly select the optimum $t_{1}^{*}$, and then obtain corresponding optimal order quantity $Q^{*}$.

\section{Numerical Examples and Sensitivity Analysis}

In order to demonstrate the procedure to obtain the optimal solution of the model, we present four examples for the model. Examples are based on piece-wise demand rate, such as $f(t)=a_{1}+b_{1} t$ and $g(t)=a_{2} e^{-b_{2} t}$.

Example 1. The parameter values of an inventory system are given as follows: $T=12$ weeks, $\mu_{1}=4$ weeks, $\mu_{2}=8$ weeks, $\alpha=0.005, \beta=2, \delta=0.04, a_{1}=30$ units, $b_{1}=5$ units, $a_{2}=100$ units, $A_{0}=\$ 500, c_{1}=\$ 2, c_{2}=\$ 3, c_{3}=\$ 12, c_{4}=\$ 8$.

Since $\Delta_{1}=18.809>0, \Delta_{2}=222.869>0$, $d^{2} \mathrm{C}_{1}\left(t_{1}\right) / d t_{1}^{2}>0$ for all $t_{1} \in\left(0, \mu_{1}\right), d^{2} \mathrm{C}_{2}\left(t_{1}\right) / d t_{1}^{2}>0$ for all $t_{1} \in\left[\mu_{1}, \mu_{2}\right)$, and $d^{2} \mathrm{C}_{3}\left(t_{1}\right) / d t_{1}^{2}>0$ for all $t_{1} \in$ $\left[\mu_{2}, T\right)$, then we get local minimum value $\mathrm{C}_{2}\left(\mu_{1}\right)=763.891$ in $\left[\mu_{1}, \mu_{2}\right)$ and $C_{3}\left(u_{2}\right)=1263.58$ in $\left[\mu_{2}, T\right)$. By solving equation $d \mathrm{C}_{1}\left(t_{1}\right) / d t_{1}=0$, we have $t_{1}^{*}=3.664$. From (10), we obtain $\mathrm{C}_{1}\left(t_{1}^{*}\right)=762.759$. Therefore, the optimal ordering quantity is $Q^{*}=637.839$ and the total average cost is $\mathrm{TC}\left(t_{1}^{*}\right)=762.759$.

Example 2. The parameter values of an inventory system are given as follows: $T=12$ weeks, $\mu_{1}=4$ weeks, $\mu_{2}=8$ weeks, $\alpha=0.005, \beta=2, \delta=0.02, a_{1}=30$ units, $b_{1}=5$ units, $a_{2}=100$ units, $A_{0}=\$ 500, c_{1}=\$ 5, c_{2}=\$ 10, c_{3}=\$ 12, c_{4}=\$ 8$.

Since $\Delta_{1}=-257.889<0, \Delta_{2}=139.269>0$, $d^{2} \mathrm{C}_{1}\left(t_{1}\right) / d t_{1}^{2}>0$ for all $t_{1} \in\left(0, \mu_{1}\right], d^{2} \mathrm{C}_{2}\left(t_{1}\right) / d t_{1}^{2}>0$ for all $t_{1} \in\left[\mu_{1}, \mu_{2}\right)$, and $d^{2} \mathrm{C}_{3}\left(t_{1}\right) / d t_{1}^{2}>0$ for all $t_{1} \in\left(\mu_{2}, T\right)$, then we get local minimum value $\mathrm{C}_{1}\left(\mu_{1}\right)=1805.883$ in $\left(0, \mu_{1}\right]$ and $C_{3}\left(u_{2}\right)=1582.32$ in $\left[\mu_{2}, T\right)$. By solving equation $d \mathrm{C}_{2}\left(t_{1}\right) / d t_{1}=0$, we have $t_{1}^{*}=6.559$. From (24), we obtain $\mathrm{C}_{2}\left(t_{1}^{*}\right)=1480.324$. Therefore, the optimal ordering quantity is $Q^{*}=571.827$ and the total average cost is $\operatorname{TC}\left(t_{1}^{*}\right)=$ 1480.324 .

Example 3. The parameter values of an inventory system are given as follows: $T=12$ weeks, $\mu_{1}=4$ weeks, $\mu_{2}=6$ weeks, $\alpha=0.005, \beta=2, \delta=0.06, a_{1}=30$ units, $b_{1}=5$ units, $a_{2}=100$ units, $A_{0}=\$ 500, c_{1}=\$ 5, c_{2}=\$ 10, c_{3}=\$ 12, c_{4}=\$ 8$.

Since $\Delta_{1}=-249.329<0, \Delta_{2}=-121.869<0$, $d^{2} \mathrm{C}_{1}\left(t_{1}\right) / d t_{1}^{2}>0$ for all $t_{1} \in\left(0, \mu_{1}\right], d^{2} \mathrm{C}_{2}\left(t_{1}\right) / d t_{1}^{2}>0$ for all $t_{1} \in\left(\mu_{1}, \mu_{2}\right]$, and $d^{2} \mathrm{C}_{3}\left(t_{1}\right) / d t_{1}^{2}>0$ for all $t_{1} \in\left[\mu_{2}, T\right)$, then we get local minimum value $\mathrm{C}_{1}\left(\mu_{1}\right)=1417.134$ in $\left(0, \mu_{1}\right]$ and $\mathrm{C}_{2}\left(u_{2}\right)=1052.016$ in $\left(\mu_{1}, \mu_{2}\right]$. By solving equation $d \mathrm{C}_{3}\left(t_{1}\right) / d t_{1}=0$, we have $t_{1}^{*}=9.120$. From (37), we obtain $\mathrm{C}_{3}\left(t_{1}^{*}\right)=889.767$. Therefore, the optimal ordering quantity is $Q^{*}=278.960$ and the total average cost is $\mathrm{TC}\left(t_{1}^{*}\right)=889.767$.

Example 4. The parameter values of an inventory system are given as follows: $T=12$ weeks, $\mu_{1}=4$ weeks, $\mu_{2}=6$ weeks, $\alpha=0.005, \beta=1.6, \delta=0.02, a_{1}=30$ units, $b_{1}=5$ units, $a_{2}=100$ units, $A_{0}=\$ 500, c_{1}=\$ 5, c_{2}=\$ 10, c_{3}=\$ 12, c_{4}=\$ 8$.

Since $\Delta_{1}=-197.263<0, \Delta_{2}=-86.675<0$, $d^{2} \mathrm{C}_{4}\left(t_{1}\right) / d t_{1}^{2}>0$ for all $t_{1} \in\left(0, \mu_{1}\right], d^{2} \mathrm{C}_{2}\left(t_{1}\right) / d t_{1}^{2}>0$ 
TABLE 1: The sensitivity of $\delta$ for the model in Example 1 .

\begin{tabular}{lccccccccc}
\hline$\delta$ & 0 & 0.01 & 0.02 & 0.03 & 0.04 & 0.05 & 0.06 & 0.07 & 0.08 \\
\hline$t_{1}^{*}$ & 3.4366 & 3.4905 & 3.5463 & 3.6042 & 3.6642 & 3.7263 & 3.7907 & 3.8574 & 3.9265 \\
$S^{*}$ & 515.0829 & 515.0829 & 511.4210 & 507.5965 & 503.6066 & 499.4433 & 495.0985 & 490.5641 & 485.8321 \\
$Q^{*}$ & 648.1034 & 648.1034 & 644.8369 & 641.4184 & 637.8392 & 634.0900 & 630.1614 & 626.0436 & 621.7267 \\
$\mathrm{TC}_{2}\left(t_{1}^{*}\right)$ & 775.2147 & 771.8437 & 768.4699 & 764.9983 & 761.4252 & 757.7473 & 753.9611 & 750.063 & 746.0498 \\
\hline
\end{tabular}

TABLE 2: The sensitivity of $\alpha$ for the models in Example 1.

\begin{tabular}{lccccccccc}
\hline$\alpha$ & 0 & 0.001 & 0.002 & 0.003 & 0.004 & 0.005 & 0.006 & 0.007 & 0.008 \\
\hline$t_{1}^{*}$ & 3.0141 & 3.1325 & 3.2569 & 3.3870 & 3.5229 & 3.6642 & 3.8105 & 3.9614 & 4.1171 \\
$S^{*}$ & 415.8882 & 432.0629 & 448.9624 & 466.5543 & 484.7907 & 503.6066 & 522.9206 & 542.6345 & 575.5923 \\
$\mathrm{Q}^{*}$ & 522.8982 & 543.9279 & 565.9745 & 589.0106 & 612.9883 & 637.8392 & 663.4729 & 689.7782 & 723.0000 \\
$\mathrm{TC}_{2}\left(t_{1}^{*}\right)$ & 638.5314 & 661.4087 & 685.1585 & 709.7639 & 735.1984 & 761.4252 & 788.3972 & 816.0570 & 845.0147 \\
\hline
\end{tabular}

TABLE 3: The sensitivity of $\beta$ for the models in Example 1.

\begin{tabular}{lccccccccc}
\hline$\beta$ & 1.4 & 1.5 & 1.6 & 1.7 & 1.8 & 1.9 & 2.0 & 2.1 & 2.2 \\
\hline$t_{1}^{*}$ & 3.1114 & 3.1995 & 3.2513 & 3.3176 & 3.40332 & 3.5148 & 3.6613 & 3.8551 & 4.1128 \\
$S^{*}$ & 427.5053 & 440.1612 & 447.1691 & 456.2029 & 467.9084 & 483.1709 & 503.6066 & 529.5334 & 564.1669 \\
$Q^{*}$ & 538.4988 & 554.7959 & 563.9494 & 575.7494 & 591.0495 & 611.0248 & 637.8392 & 672.0262 & 717.9964 \\
$\mathrm{TC}_{2}\left(t_{1}^{*}\right)$ & 644.4596 & 670.3559 & 680.1679 & 692.9359 & 709.5918 & 731.4743 & 761.4252 & 797.6508 & 845.0147 \\
\hline
\end{tabular}

for all $t_{1} \in\left(\mu_{1}, \mu_{2}\right]$, and $d^{2} \mathrm{C}_{3}\left(t_{1}\right) / d t_{1}^{2}>0$ for all $t_{1} \in$ $\left[\mu_{2}, T\right)$, then we get local minimum value $\mathrm{C}_{1}\left(\mu_{1}\right)=1252.59$ in $\left(0, \mu_{1}\right]$ and $C_{2}\left(u_{2}\right)=1052.016$ in $\left(\mu_{1}, \mu_{2}\right]$. By solving equation $d \mathrm{C}_{3}\left(t_{1}\right) / d t_{1}=0$, we have $t_{1}^{*}=8.627$. From (37), we obtain $\mathrm{TC}\left(t_{1}^{*}\right)=842.935$. Therefore, the optimal ordering quantity is $Q^{*}=241.524$ obtained from (42), and the minimum cost $\mathrm{TC}\left(t_{1}^{*}\right)=842.935$.

In order to clearly indicate the effects of parameters such as $\delta, \alpha$, and $\beta$ on $S^{*}, Q^{*}$, and $\mathrm{TC}_{i}\left(t_{1}^{*}\right)(i=1,2)$, respectively, we study the sensitivity of different parameters under the studied inventory model. The sensitivity analysis is performed on the base of Example 1, and the results are shown in Tables $1-3$, respectively.

From Table 1, we observe that inventory level $S^{*}$, order quantity $Q^{*}$, and the total average cost $\mathrm{TC}_{i}\left(t_{1}^{*}\right)(i=1,2)$ gradually decrease as the shortage parameter $\delta$ increases for the model, and the replenishment time $t_{1}^{*}$ increases as $\delta$ increases for the model starting with shortage. Also, we observe that the values of $t_{1}^{*}, S^{*}, Q^{*}$, and TC $\left(t_{1}^{*}\right)$ are slightly sensitive to the changes of $\delta$.

From Table 2, we find that when the deterioration parameter $\alpha$ increases, then $S^{*}, Q^{*}$, and $\operatorname{TC}\left(t_{1}^{*}\right)$ increase for both models, and the replenishment time $t_{1}^{*}$ increases for the model starting with shortage. We see that the values of $t_{1}^{*}, S^{*}$, $Q^{*}$, and $\mathrm{TC}\left(t_{1}^{*}\right)$ all are temperately sensitive to the changes of $\alpha$ for the model.

From Table 3, we find that $S^{*}, Q^{*}$, and $\mathrm{TC}\left(t_{1}^{*}\right)$ increase as the deterioration parameter $\beta$ increases for both models. Also, we find that the replenishment time $t_{1}^{*}$ increases as $\beta$ increases for the model starting with shortage. We see that the values of $t_{1}^{*}, S^{*}, Q^{*}$, and $\mathrm{TC}\left(t_{1}^{*}\right)$ all are moderately sensitive to the changes of $\beta$ for the model.

\section{Conclusion}

This paper studies an inventory model for Weibulldistributed deterioration with trapezoidal type demand rate and partial backlogging. First, an optimal replenishment strategy for deteriorating items with the model starting with shortage is proposed. Then, numerical examples are provided to illustrate the theoretical results. From the analysis of Tables $1-3$, it can be found that the replenishment time point $t_{1}^{*}$, order quantity $Q^{*}$, and the total average cost $\mathrm{TC}\left(t_{1}^{*}\right)$ are moderately sensitive to the changes of $\alpha$ and $\beta$ and lowly sensitive to the changes of $\delta$, respectively. However, if the order cycle $(T)$ is a decision variable, such inventory model will be of more practical significance, which provides us an interesting topic in future.

\section{Competing Interests}

The author declares that there are no competing interests.

\section{Acknowledgments}

The research is supported partly by National Natural Science Foundation of China (Grant no. 71502100).

\section{References}

[1] P. M. Ghare and G. F. Schrader, "A model for exponentially decaying inventories," Journal of Industrial Engineering, vol. 14, no. 3, pp. 238-243, 1963.

[2] R. P. Covert and G. C. Philip, "An EOQ model for items with weibull distribution deterioration," AIIE Transaction, vol. 5, no. 4, pp. 323-326, 1973. 
[3] H.-M. Wee, J. C. P. Yu, and S. T. Law, “Two-warehouse inventory model with partial backordering and Weibull distribution deterioration under inflation," Journal of the Chinese Institute of Industrial Engineers, vol. 22, no. 6, pp. 451-462, 2005.

[4] G. K. Yang, R. Lin, J. Lin, K.-C. Hung, P. Chu, and W. Chouhuang, "Note on inventory models with Weibull distribution deterioration," Production Planning \& Control, vol. 22, no. 4, pp. 437-444, 2011.

[5] N. H. Shah, H. N. Soni, and K. A. Patel, "Optimizing inventory and marketing policy for non-instantaneous deteriorating items with generalized type deterioration and holding cost rates," Omega, vol. 41, no. 2, pp. 421-430, 2013.

[6] C.-T. Yang, L.-Y. Ouyang, H.-F. Yen, and K.-L. Lee, "Joint pricing and ordering policies for deteriorating item with retail pricedependent demand in response to announced supply price increase," Journal of Industrial and Management Optimization, vol. 9, no. 2, pp. 437-454, 2013.

[7] R. M. Hill, "Inventory models for increasing demand followed by level demand," Journal of the Operational Research Society, vol. 46, no. 10, pp. 1250-1259, 1995.

[8] B. Mandal and A. K. Pal, "Order level inventory system with ramp type demand rate for deteriorating items," Journal of Interdisciplinary Mathematics, vol. 1, no. 1, pp. 49-66, 1998.

[9] K.-S. Wu, "An EOQ inventory model for items with Weibull distribution deterioration, ramp type demand rate and partial backlogging," Production Planning \& Control, vol. 12, no. 8, pp. 787-793, 2001.

[10] K. Skouri, I. Konstantaras, S. Papachristos, and I. Ganas, "Inventory models with ramp type demand rate, partial backlogging and Weibull deterioration rate," European Journal of Operational Research, vol. 192, no. 1, pp. 79-92, 2009.

[11] Y. Tan and M. X. Weng, "A discrete-in-time deteriorating inventory model with time-varying demand, variable deterioration rate and waiting-time-dependent partial backlogging," International Journal of Systems Science, vol. 44, no. 8, pp. 14831493, 2013.

[12] S. Pal, G. S. Mahapatra, and G. P. Samanta, "A production inventory model for deteriorating item with ramp type demand allowing inflation and shortages under fuzziness," Economic Modelling, vol. 46, no. 2, pp. 334-345, 2015.

[13] M. A. Ahmed, T. A. Al-Khamis, and L. Benkherouf, "Inventory models with ramp type demand rate, partial backlogging and general deterioration rate," Applied Mathematics and Computation, vol. 219, no. 9, pp. 4288-4307, 2013.

[14] S. Agrawal, S. Banerjee, and S. Papachristos, "Inventory model with deteriorating items, ramp-type demand and partially backlogged shortages for a two warehouse system," Applied Mathematical Modelling, vol. 37, no. 20-21, pp. 8912-8929, 2013.

[15] T. Singh and H. Pattnayak, "An EOQ inventory model for deteriorating items with varying trapezoidal type demand rate and Weibull distribution deterioration," Journal of Information and Optimization Sciences, vol. 34, no. 6, pp. 341-360, 2013.

[16] J. Wu, L.-Y. Ouyang, L. E. Cárdenas-Barrón, and S. K. Goyal, "Optimal credit period and lot size for deteriorating items with expiration dates under two-level trade credit financing," European Journal of Operational Research, vol. 237, no. 3, pp. 898-908, 2014.

[17] M. Rabbani, N. P. Zia, and H. Rafiei, "Coordinated replenishment and marketing policies for non-instantaneous stock deterioration problem," Computers \& Industrial Engineering, vol. 88, pp. 49-62, 2015.
[18] M. B. Cheng and G. Q. Wang, "A note on the inventory model for deteriorating items with trapezoidal type demand rate," Computers \& Industrial Engineering, vol. 56, no. 4, pp. 12961300, 2009.

[19] M. B. Cheng, B. X. Zhang, and G. Q. Wang, "Optimal policy for deteriorating items with trapezoidal type demand and partial backlogging," Applied Mathematical Modelling, vol. 35, no. 7, pp. 3552-3560, 2011.

[20] R. Uthayakumar and M. Rameswari, "An economic production quantity model for defective items with trapezoidal type demand rate," Journal of Optimization Theory and Applications, vol. 154, no. 3, pp. 1055-1079, 2012.

[21] K.-P. Lin, "An extended inventory models with trapezoidal type demands," Applied Mathematics and Computation, vol. 219, no. 24, pp. 11414-11419, 2013.

[22] J. Lin, K.-C. Hung, and P. Julian, “Technical note on inventory model with trapezoidal type demand," Applied Mathematical Modelling, vol. 38, no. 19-20, pp. 4941-4948, 2014.

[23] L. Zhao, "An inventory model under trapezoidal type demand, Weibull-distributed deterioration, and partial backlogging," Journal of Applied Mathematics, vol. 2014, Article ID 747419, 10 pages, 2014. 


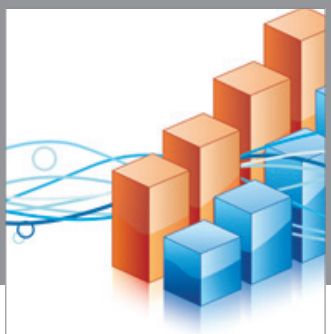

Advances in

Operations Research

vatem alat4

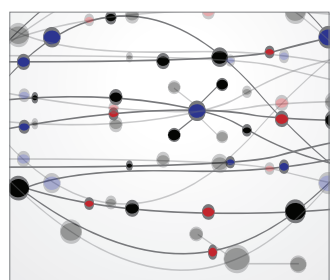

\section{The Scientific} World Journal
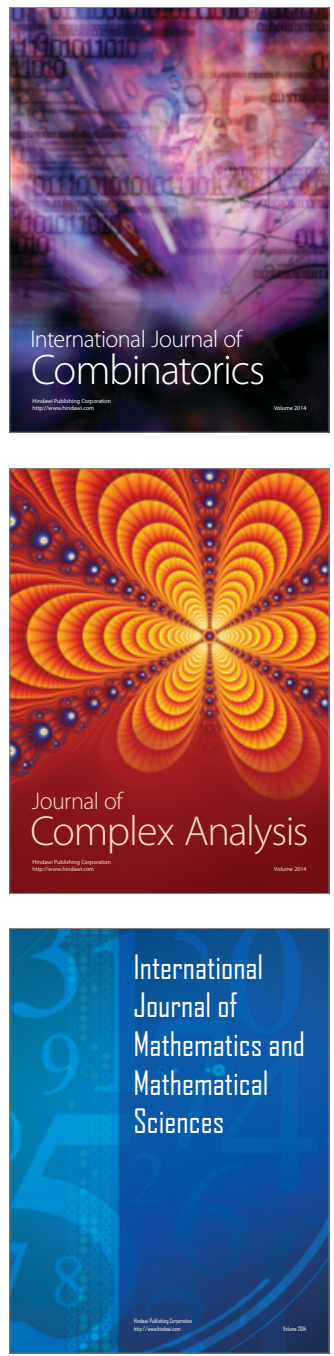
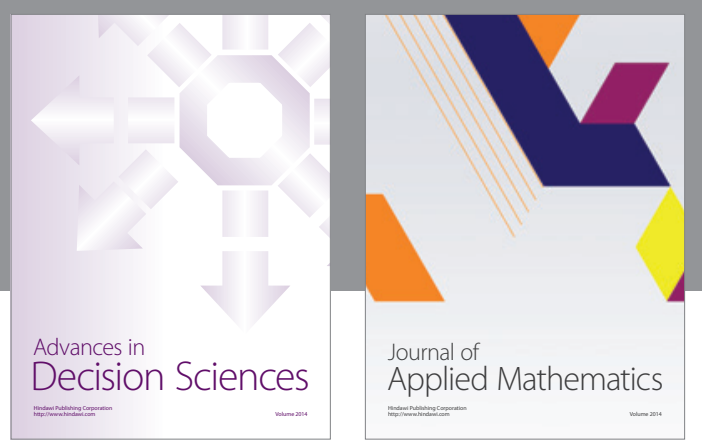

Algebra

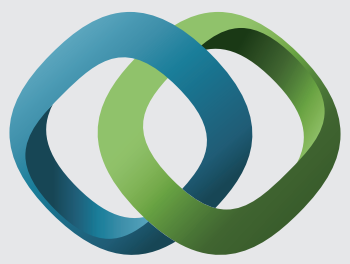

\section{Hindawi}

Submit your manuscripts at

http://www.hindawi.com
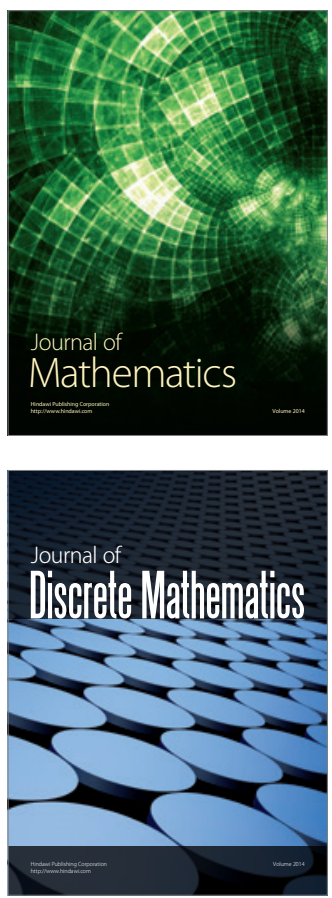

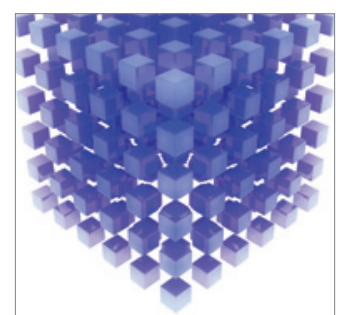

Mathematical Problems in Engineering
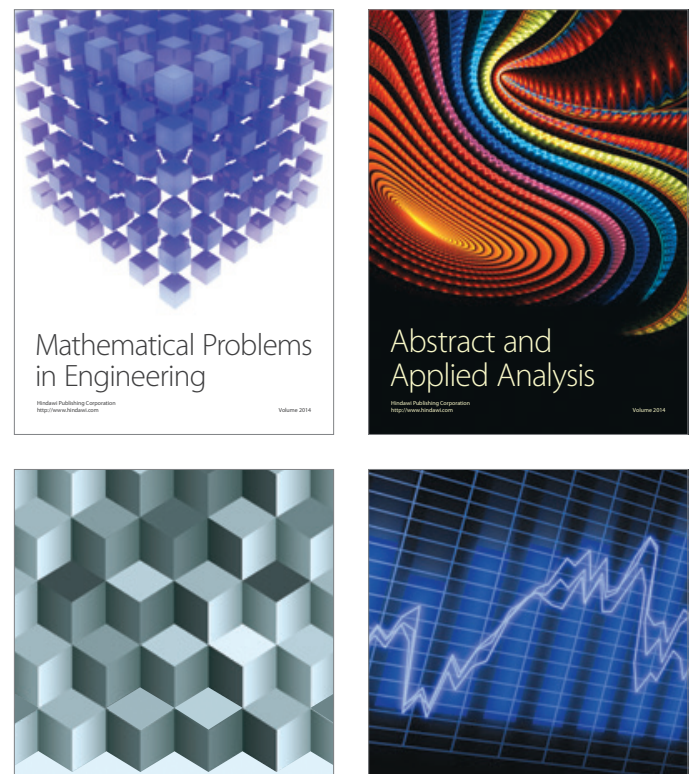

Journal of

Function Spaces

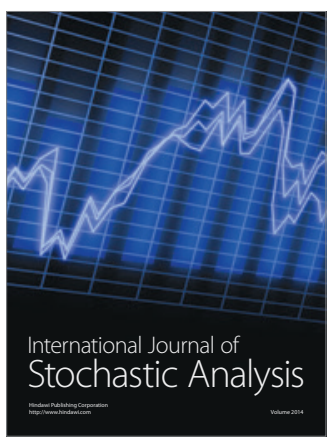

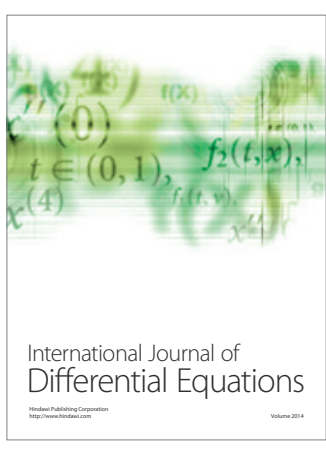
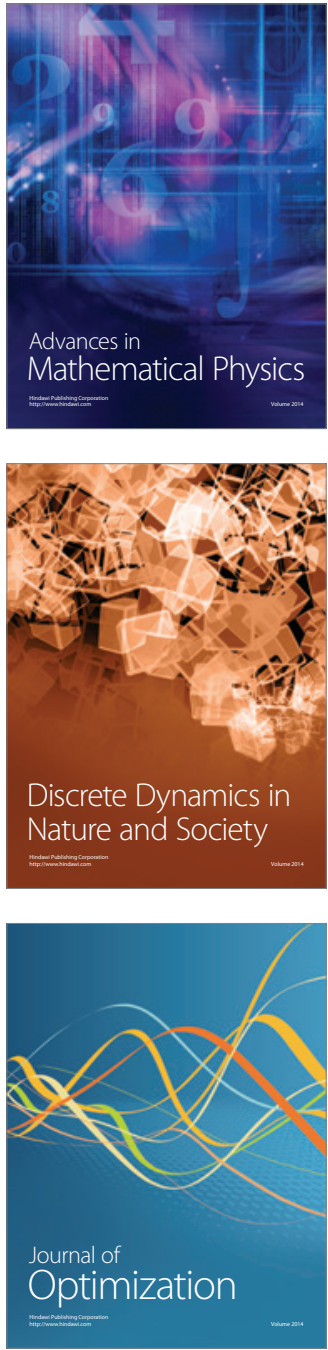